九州大学学術情報リポジトリ

Kyushu University Institutional Repository

\title{
STATISTICAL INFERENCE PROCESSES AND THEIR PROPERTIES BASED ON KNOWLEDGE FUNCTIONS IN NISAN SYSTEM
}

Kurihara, Koji

Department of Statistics, College of Liberal Arts and Sciences, Okayama University

https://doi.org/10.5109/13393

出版情報: Bulletin of informatics and cybernetics. 23 (1/2), pp.41-54, 1988-03. Research Association of Statistical Sciences

バージョン：

権利関係 : 


\title{
STATISTICAL INFERENCE PROCESSES AND THEIR PROPERTIES BASED ON KNOWLEDGE FUNCTIONS IN NISAN SYSTEM
}

\author{
By
}

\author{
Koji KURIHARA*
}

\begin{abstract}
Successive processes of statistical methods are usually applied to data analysis in practice. Excepting some studies of prototypes, however, mathematical properties of such many inference processes are still remained unclear, although their investigations are strongly needed in routine. In the present paper, inference processes are generally classified to three categories, and several plans proposed by previous authors are extended with wide flexibility, and are discussed on their properties. For their numerical evaluation, knowledge functions developed in NISAN system are applied.
\end{abstract}

\section{Introduction}

Theories of serial application of statistical methods have been studied in the fields of multistage sampling inspection plans, sequential analyses of statistical inference, successive processes of statistical methods, and so on. Since such serial procedures for inference are very attractive in view of sample size, sampling cost, significance level, power and relative efficiency of the overall process of tests, and the performed precision for estimation of parameters, there exist still various schemes to be investigated, excepting rather simple procedures already discussed by many authors, e.g. Kitagawa [8], Wald [14], Asano et al. [2, 3], McPherson and Armitage [10], Miller [11], Aroian [1], Pocock [13], Gould and Pecore [7] and so on. Regarding such inference procedures, there exist three categories, depending on the aim of actual process of statistical recognition [2]. The first kind of categories is process via identical tests by adding samples, the second is process via different tests with a similar purpose, and the third is inference process via different tests with different purposes. The respective categories contain two types, say Types 1 and 2 shown in Figures 1 and 2, with one-by-one, partial, group or adaptive sampling rule. In the present paper, several serial processes of inference are extended theoretically and their numerical evaluations are precisely investigated about three categories. For these evaluations, knowledge functions in NISAN system are effectively used. That is to say, PRTP command gives the power of an overall test process, expected number of samples (ASN) and expected cost (AC), which consists in both costs of sampling set up and samples, for the respective sampling

\footnotetext{
* Department of Statistics, College of Liberal Arts and Sciences, Okayama University, Japan.
} 


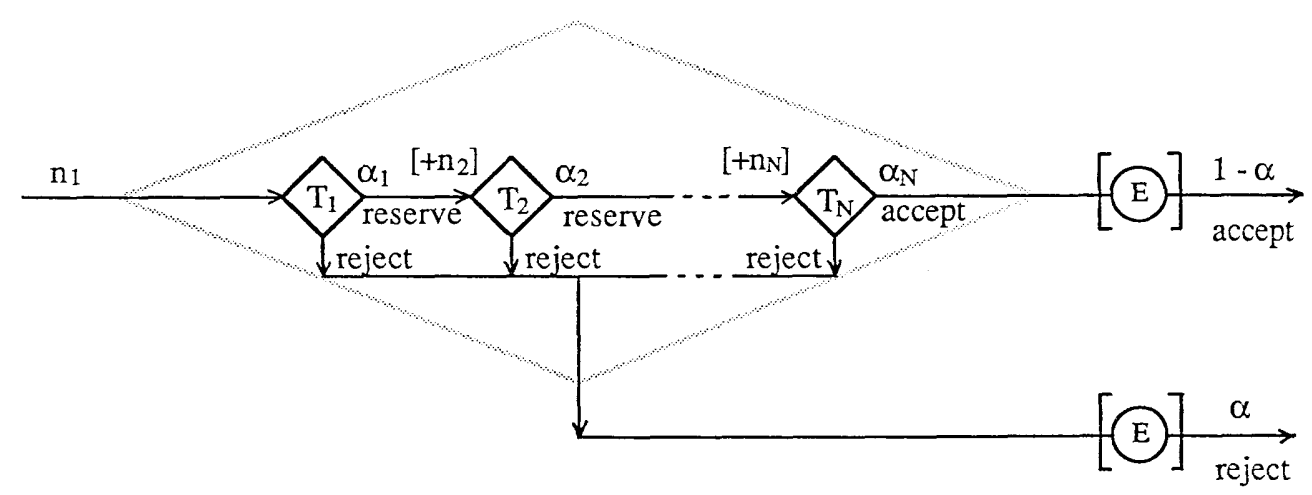

Figure $1 \quad N$-stage test process plan of Type 1.

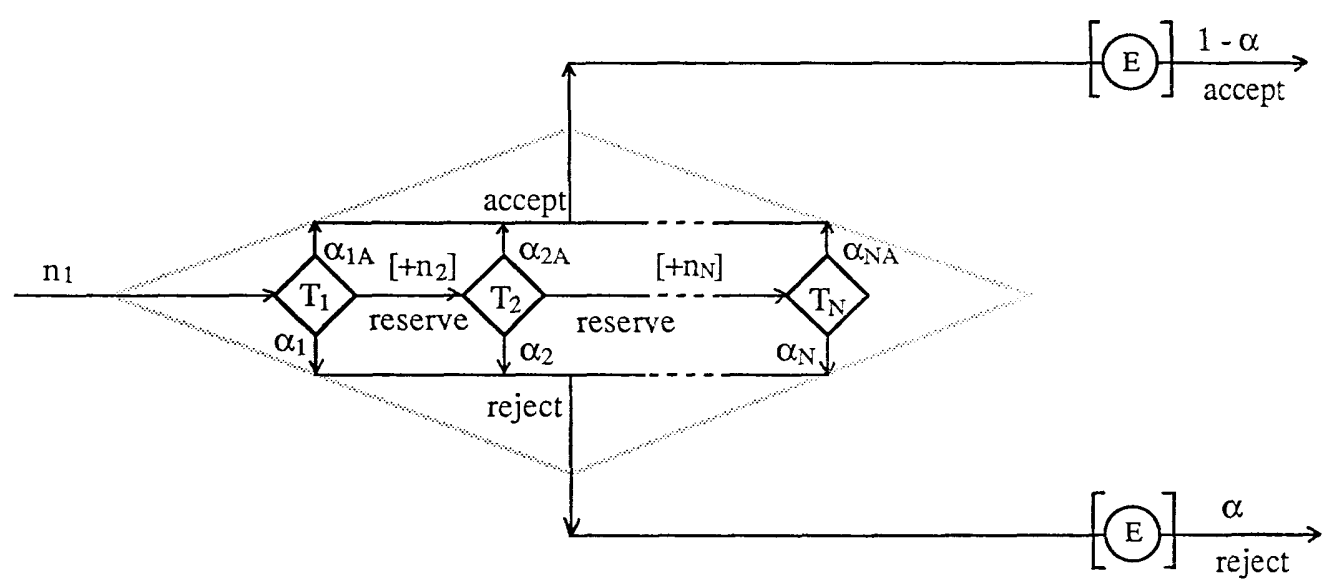

Figure $2 N$-stage test process pian of Type 2.

rules. PRTE command gives estimation of parameters, mean square error, and value of risk function, basing on an test procedure. PRDIST command is to give a nominal significance level for a conditional distribution of the final test statistics due to a test process. Such PR-family of commands in NISAN system is possible to examine properties of about 90 papers in the recent 300 papers related to inference processes.

\section{Fundamental Properties of Statistical Inference Processes}

Let $\boldsymbol{X}_{i}=\left(X_{i 1}, X_{i 2}, \cdots, X_{i n_{i}}\right)$ be a random variable vector with $n_{i}$ samples, and let $T_{i}\left(\boldsymbol{X}_{\boldsymbol{i}}\right)$ be a statistic at $i$-th stage, $i=1,2, \cdots, N$.

Definition 1. $N$ stage process with an overall significance level $\alpha$ is defined as follows,

$$
\operatorname{Pr}\left[\bigcup_{i \in I}\left(T_{i}\left(\boldsymbol{X}_{i}\right) \in C_{i} \mid H_{0 i}\right)\right] \leqq \alpha,
$$

where $C_{i}$ is a critical region of $i$-th test for a null hypothesis $H_{0 i}, i=1,2, \cdots, N$, and $I$ is a non-empty index set $I \subset[1,2, \cdots, N]$. 
Let $\alpha_{i}$ be a nominal significance level for critical region $C_{i}$ and let $\alpha_{i A}$ a nominal acceptance probability for acceptance region $A_{i}, i=1,2, \cdots N$. Let $\operatorname{Pr}\left(C_{i}\right)$ and $\operatorname{Pr}\left(A_{i}\right)$ denote $\operatorname{Pr}\left(T_{i}\left(\boldsymbol{X}_{\boldsymbol{i}}\right) \in C_{i}\right)$ and $\operatorname{Pr}\left(T_{i}\left(\boldsymbol{X}_{\boldsymbol{i}}\right) \in A_{i}\right)$, respectively, in short. The following Lemma 1 is led by Bonferroni's inequality.

Lemma 1. Let $T$ be an $N$ stage test process with $\alpha_{i}=\alpha / N, i=1,2, \cdots, N$. Then, the overall significance level of $T$ is less or equal to $\alpha$

Proof. By using additive theorem of probability, the lemma is derived.

Lemma 2. Let $T_{1}$ and $T_{2}$ be $N$ stage proecss of Type 1 and Type 2, respectively, with the same critical region $C_{i}, i=1,2, \cdots, N$. Then, the overall significance level of $T_{1}$ is greater than or equal to $T_{2}$.

PROOF.

$$
\begin{aligned}
\alpha\left(T_{1}\right) & =\operatorname{Pr}\left(\bigcup_{i \in I} C_{i}, T_{1}\right)=1-\operatorname{Pr}\left(\bigcup_{i \in I} A_{i} \mid T_{1}\right)=1-\operatorname{Pr}\left(A_{.} \mid T_{1}\right) \\
& \geqq 1-\operatorname{Pr}\left(\bigcup_{i \in I} A_{i} \mid T_{2}\right)=\operatorname{Pr}\left(\bigcup_{i \in I} C_{i} \mid T_{2}\right)=\alpha\left(T_{2}\right),
\end{aligned}
$$

where $I$ is index set defined in Definition 1.

Usually, evaluation for the process with $\alpha_{i}$ by Lemma 1 is conservative, so that exact evaluation for overall significance level is to be calculated.

For $N$ stage test process of Type 1, the overall hypothesis $H_{0}$ is rejected in case that at least one stage is rejected in $N$ stages with nominal significance level $\alpha_{i}, i=$ $1,2, \cdots, N$.

On the other hand, there are $2^{N}$ patterns in the $N$ stage test process basing on significance and nonsignificance patterns, In the process of Type 1 , the overall $\alpha$ is given by the sum of probabilities for $2^{N}-1$ patterns under a null hypothesis, excepting the probabilities of $N$ nonsignificances. Thus, the following theorem is derived.

THEOREM 1. Let $T$ be an $N$ stage test process in which the overall hypothesis $H_{0}$ is rejected in case that at least $k$-th stage, $k=0,1, \cdots, N$, is rejected in $N$ stages with nominal significance level $\alpha_{i}, i=1,2, \cdots, N$, and tests at each stage are independent each other, then the overall significance level $\delta$ for $T$ is given by

$$
\delta=\sum_{i=k}^{N} \prod_{\substack{\omega_{i} \subset \Omega, j \in \omega_{i}}}\left[\alpha_{j} \prod_{t \in \Omega-\omega_{i}}\left(1-\alpha_{t}\right)\right]
$$

where $\Omega$ is a set $[1,2, \cdots, N]$ and $\omega_{i}$ is the subset of $\Omega$.

PROOF. Under the independence of each stages, the probability that the $H_{0}$ is rejected at $k$-th stage in $N$ stages plan is given by

$$
\prod_{j \in \omega_{k}}\left[\alpha_{j} \prod_{t \in \Omega-\omega_{k}}\left(1-\alpha_{t}\right)\right] .
$$

Thus, the probability for rejecting at least $k$-th stage in $N$ stages is given by the sum of probabilities up to the $N$ stages.

COROLLARY 1. Under the condition of Theorem 1 , if each nominal signifucance level is equals to $\alpha^{*}$, the overall significance level is given by the sum of binomial $B\left(N, \alpha^{*}\right)$ probabilities up to $\mathrm{N}$-th stage from $k$-th stage.

Proof. Every $i$-th stage, $i=1,2, \cdots, N$, independently has the same probability $\alpha^{*}$ for reject, so that the desired overall significance level is given by the upper probability 
of binomial distribution with parameters, $N$ and $\alpha^{*}$.

In the actual situations, however, the tests at each stage have some associations, so that the decision for the overall $\alpha$ with nominal $\alpha_{i}$ is a difficult problem.

For the power, ASN and AC, it is usually very laborious and complicated to obtain numerically theoretical values for the conditional distribution of test statistics in test processes, based on mathematical formulations. For certain practical situations, asymptotic theories are applied. In the case of small sample size or closed plan, however, direct evaluation is to be preferred.

\section{Inference Processes via Identical Tests}

The inference processes in this section are the processes via identical tests, for instance, repeated significance tests (RST) and sequential probability ratio tests (SPRT). These inference processes are most popular, in which one statistical test is repeatedly applied by adding samples at every stage, when any decision is reserved. It is laborious and complicated to obtain numerical values of probability for the mathematical properties of multi-stage procedure. In the case of small sample size, however, direct methods for evaluation is to be preferred. In this section, two direct methods for closed SPRT and RST are evaluated.

\subsection{Direct method for normal mean test}

Aroian [1] presented a method of numerical evaluation of the power and ASN for SPRT one sample mean test $(T)$ of a closed process. That is, let $T$ be a one sample normal mean test of a closed type at $N$ samples from a normal population $N\left(\mu, \sigma^{2}\right), \sigma^{2}$ : known, based on one by one sampling SPRT. The hypothesis is $H_{0}: \mu=\mu_{0}$ against the alternative $H_{1}: \mu=\mu_{1}$ and the critical value at $i$-th stage, $i=1,2, \cdots, N-1$, is used the value for SPRT. Then, the procedure of $T$ is shown in the following way.

PROCEDURE 1. At $i$-th stage, $1 \leqq i \leqq N-1$,

$$
\begin{array}{lll}
\text { accept } H_{0}, & \text { if } & \sum_{j=1}^{i} x_{j} \geqq X_{i 1}, \\
\text { accept } H_{1}, & \text { if } & \sum_{j=1}^{i} x_{j} \leqq X_{i 0}, \\
\text { continue sampling, } & \text { otherwise, }
\end{array}
$$

the final stage, i.e. $i=N$

where

$$
\begin{array}{lll}
\text { accept } H_{0}, & \text { if } & \sum_{j=1}^{i} x_{j} \geqq\left(X_{i 0}+X_{i 1}\right) / 2, \\
\text { accept } H_{1}, & \text { otherwise, }
\end{array}
$$

$$
\begin{aligned}
& X_{i j}=\sigma^{2} /\left(\mu_{1}-\mu_{0}\right) \log A_{j}+i\left(\mu_{1}-\mu_{0}\right) / 2, \\
& A_{0}=\beta /(1-\alpha), \quad A_{1}=(1-\beta) / \alpha, \quad(i=1,2, \cdots, N),(j=0,1) .
\end{aligned}
$$

The mathematical formulations of the power, ASN and $\mathrm{AC}$ for this process are shown as below.

Theorem 2. Let $T$ be an $N$ stage test process which has Procedure 1. Then, power, 
ASN and AC are given by the foliowing formulae respectively, where $K_{1}$ and $K_{2}$ are sample and setup costs.

$$
\begin{aligned}
& L(\mu)=\sum_{i=1}^{N} C^{i}\left[\int_{-\infty}^{L_{i}-Y_{i-1}} \cdots \int_{L_{1}}^{U_{1}} f_{i}(\mu) \prod_{j=1}^{i} d x_{j}\right] \\
& E_{1}(\mu)=\sum_{i=1}^{N} i C^{i}\left[\int_{U_{i}-Y_{i-1}}^{\infty}+\int_{-\infty}^{L_{i}-Y_{i}-1}\right] \int_{L_{i-1}-Y_{i-2}}^{U_{i-1}-Y_{i-2}} \cdots \int_{L_{1}}^{U_{1}} f_{i}(\mu) \prod_{j=1}^{i} d x_{j} \\
& E_{2}(\mu)=K_{1} \sum_{i=1}^{N-1} C^{i}\left[\int_{L_{i}-Y_{i-1}}^{U_{i}-Y_{i-1}} \cdots \int_{L_{1}}^{U_{1}} f_{i}(\mu) \prod_{j=1}^{i} d x_{j}\right]+K_{2} E_{1}(\mu)
\end{aligned}
$$

where

$$
\begin{aligned}
& f_{i}(\mu)=\exp \left[-\sum_{j=1}^{i}\left(x_{j}-\mu\right)^{2} /\left(2 \sigma^{2}\right)\right], \quad \int_{L_{0}}^{U_{0}} f_{0}(\mu) d x_{0}=1, \\
& C=(\sqrt{2 \pi} \sigma)^{-1}, \quad Y_{i}=\sum_{j=1}^{i} x_{j}, \quad U_{i}=X_{i 1}, \quad L_{i}=X_{i 0} .
\end{aligned}
$$

Proof. The probability of accept $H_{1}$ at $i$-th stage is given by the conditional probability

$$
C^{i}\left[\int_{-\infty}^{L_{i}-Y_{i}-1} \cdots \int_{L_{1}}^{U_{1}} f_{i}(\mu) \prod_{j=1}^{i} d x_{j}\right]
$$

and power is shown the sum of these probabilities up to $N$ stage. The ASN is given the product of the probability for accept $H_{0}$ or $H_{1}$ and sample size when the process terminates, and AC is sum of ASN multiplied by sample cost and average stage by set up cost.

The numerical evaluation of these values are very complicated so that Aroian evaluated only the power and ASN for $\alpha=\beta=0.09975, N=7, \mu_{0}=-\mu_{1}=0.5$, shown in left side of Table 1 . The extended results including standard deviation of ASN are shown in right side by PRTP command in knowledge function with good precision. Note that this process is a one by one sampling, so that the average cost is simply $\left(K_{1}+K_{2}\right)$ multiplied by ASN, where $K_{1}$ and $K_{2}$ are sample and setup costs, respectively.

Table 1 Properties of closed SPRT for normal mean test.

\begin{tabular}{|l|ll|llll|}
\hline \multirow{2}{*}{$\mu$} & \multicolumn{2}{|c|}{ L. Aroian (1968) } & \multicolumn{4}{c|}{ PRTP Command (NISAN) } \\
\cline { 2 - 7 } & O.C. & A.S.N. & O.C. & A.S.N. & S.N.D. & Cost* \\
\hline 0.75 & 0.971 & 3.652 & 0.972 & 3.662 & 1.789 & 14.648 \\
$0.50\left(\mu_{0}\right)$ & 0.898 & 4.391 & 0.903 & 4.395 & 1.981 & 17.580 \\
0.25 & 0.739 & 5.012 & 0.734 & 5.010 & 1.986 & 20.040 \\
0.00 & 0.506 & 5.266 & 0.498 & 5.231 & 1.956 & 20.924 \\
-0.25 & 0.262 & 5.012 & 0.266 & 5.009 & 1.992 & 20.036 \\
$-0.50\left(\mu_{1}\right)$ & 0.102 & 4.391 & 0.101 & 4.378 & 1.986 & 17.512 \\
-0.75 & 0.029 & 3.652 & 0.031 & 3.629 & 1.803 & 14.516 \\
\hline
\end{tabular}

* $\mathrm{K}_{1}=1, \mathrm{~K}_{2}=3$ 
For the extension of such linds of investigation, let a process be considered for a mixed distribution $G(x)=1-p F\left(x, \mu_{1}, \sigma^{2}\right)+p F\left(x, \mu_{1}, \sigma^{2}\right.$, which has the same null, $p=0$ and alternative, $p=1$, hypotheses of Procedure 1. The distribution of mean for sample size $n$ is not normal witi mean, $E(X)=1-p \mu_{0}+p \mu_{1}$, and variance, $V(X)=$ $\left[\left(\mu_{0}-\mu_{1}\right)^{2} p(1-p)+\sigma^{2}\right] ;$. The power, ASN and $A C$ for this process are given in the following way.

THEOREM 3. Let $T$ be an N-stage one sample nomal mean test from mixed distribution $G(x)=(1-p) F\left(x \mid \mu_{0}, \sigma^{2}\right)+p F\left(x, \mu_{2}, \sigma^{2}\right), \sigma^{2}$ : known, based on one by one sampling SPRT for $H_{0}: p=0$ against $H_{1}: p=1$. Then, the pouer, ASN and AC are given by

$$
\begin{aligned}
& L(p)=\sum_{i=1}^{N} C^{i}\left[\int_{-\infty}^{L_{i}-Y_{i-1}} \cdots \int_{L_{1}}^{U_{1}} \prod_{j=1}^{i} g_{j} p, \prod_{j=1}^{i} d x_{j}\right] \\
& \left.E_{1}(p)=\sum_{i=1}^{N} i C^{i}\left[\int_{U_{i-Y_{i-1}}}^{\infty}+\int_{-\infty}^{L_{i}-Y_{i-1}}\right]\right]_{L_{i-1}-Y_{i-2}}^{U_{i-1}-Y_{i-2}} \cdots \int_{L_{1}}^{U_{1}} \prod_{j=1}^{i} g_{j}(p) \prod_{j=1}^{i} d x_{j}, \\
& E_{2}(p)=K_{1} \sum_{i=1}^{N-1} C^{i}\left[\int_{L_{i}-Y_{i-1}}^{U_{i}-Y_{i-1}} \ldots \int_{L_{1}}^{U_{1}} \prod_{j=1}^{i} g_{j}(p) \prod_{j=1}^{i} d x_{j}\right]+K_{2} E_{1}(p),
\end{aligned}
$$

respectively, where

$$
\begin{aligned}
& g_{i}(p)=(1-p) \exp \left[-\left(x_{j}-\mu_{0}\right)^{2} /\left(2 \sigma^{2}\right)\right]+D \exp \left[-\left(x_{j}-\mu_{1}\right)^{2} /\left(2 \sigma^{2}\right)\right], \\
& \int_{L_{0}}^{U_{0}} g_{0}(p) d x_{0}=1, \quad C=(\sqrt{2 \pi} \sigma)^{-1}, \quad Y_{i}=\sum_{j=1}^{i} x_{j}, \\
& U_{i}=X_{i 1}, \quad L_{i}=X_{i 0}, \quad X_{i j}=\sigma^{2} /\left(\mu_{1}-\mu_{0}\right) \log A_{j}+i\left(\mu_{1}-\mu_{0}\right) / 2, \\
& A_{0}=\beta /(1-\alpha), \quad A_{1}=(1-\beta) / \alpha \quad(i=1,2, \cdots, N),(j=0,1) .
\end{aligned}
$$

Proof. The probability of accept $H_{1}$ at $i$-th stage is given by the conditional probability of the mixed distribution

$$
C^{i}\left[\int_{-\infty}^{L_{i}-Y_{i-1}} \cdots \int_{L_{1}}^{U_{1}} \prod_{j=1}^{i} g_{j}(p) \prod_{j=1}^{i} d x_{j}\right]
$$

and power, ASN and $\mathrm{AC}$ are given similarly by the proof of Theorem 2 .

The numerical evaluations for this process are shown in Table 2 with ASN's stan-

Table 2 Power and ASN for extended mixed alternatives.

\begin{tabular}{|l|l|l|l|}
\hline $\mathrm{p}$ & $\mathrm{OC}$ & $\mathrm{ASN}$ & $\mathrm{SD}$ \\
\hline $0.0 *$ & $0.898^{*}$ & $4.391 *$ & 1.981 \\
0.1 & 0.834 & 4.541 & 2.016 \\
0.2 & 0.754 & 4.685 & 2.051 \\
0.3 & 0.671 & 4.763 & 2.060 \\
0.4 & 0.592 & 4.892 & 2.074 \\
0.5 & 0.491 & 4.927 & 2.080 \\
0.6 & 0.412 & 4.851 & 2.067 \\
0.7 & 0.324 & 4.783 & 2.072 \\
0.8 & 0.243 & 4.707 & 2.049 \\
0.9 & 0.162 & 4.559 & 2.011 \\
$1.0 *$ & $0.102 *$ & $4.391 *$ & 1.986 \\
\hline
\end{tabular}


dard deviation by MIXRAN and PRTP commands, where MIXRAN is to give the mixed distribution.

\subsection{Direct method for binomial case}

Colton-McPherson [4] proposed a two stage group sampling process from binomial distribution $B(n, p)$, and gave the significance level, power and ASN considering the loss, where $p$ is the probability of success defined in a single trial. Suppose that an extended process $T$ be an $N$ stage group sampling test process of Type 2 for binomial distribution $B\left(n_{i}, p\right), i=1,2, \cdots, N$, and a hypothesis is $H_{0}: p=p_{0}$ against $H_{1}: p=p_{1}$. Then, the process $T$ is shown in the following way.

Procedure 2. At $i$-th stage, $1 \leqq i \leqq N-1$,

$$
\begin{array}{lll}
\text { accept } H_{0}, & \text { if } & \sum_{j=1}^{i} r_{j}>C_{N}, \\
\text { accept } H_{1}, & \text { if } & \sum_{j=1}^{i} r_{j} \leqq C_{i}, \\
\text { continue sampling, } & \text { otherwise, }
\end{array}
$$

the final stage, i.e. $i=N$

$$
\begin{array}{ll}
\text { accept } H_{0}, & \text { if } \\
\text { accept } H_{1}, & \sum_{j=1}^{i} r_{j}>C_{N},
\end{array}
$$

where $C_{i}$ : critical value of $i$-th stage, $r_{i}$ : number of successes, $i=1,2, \cdots, N$.

THEOREM 4. Let $T$ be an $N$ stage test process which has Procedure 2 . Then, the power, ASN and AC are given by

$$
\begin{aligned}
& L(p)=1-\sum_{i=1}^{N} \sum_{j=C_{i-1}+1}^{C_{i}} h_{i-1}\left(k_{i-1}\right) G\left(n_{i}, C_{i-j}, p\right), \\
& E_{1}(p)=\sum_{i=1}^{N}\left\{n_{i}\left[\sum_{k_{i}=C_{i-1}+1}^{C_{N}} h_{i-1}\left(k_{i}\right)\right]\right\}, \\
& E_{2}(p)=K_{1} \sum_{i=0}^{N-1} \sum_{k}^{C_{i}} \sum_{C_{i+1}} h_{i}\left(k_{i}\right)+K_{2} E_{1}(p),
\end{aligned}
$$

where

$$
\begin{aligned}
& g(n, j, p)= \begin{cases}\left(\begin{array}{c}
n \\
j
\end{array}\right) p^{j}(1-p)^{n-j} & 0 \leqq j \leqq n \\
0 & \text { otherwise }\end{cases} \\
& G(n, c, p)= \begin{cases}\sum_{j=0}^{c} g(n, j, p) & 0 \leqq j \leqq n \\
0 & \text { otherwise, }\end{cases} \\
& h_{i}\left(k_{i}\right)= \begin{cases}\sum_{k_{i}-1=c_{i-1}+1}^{k_{i}} h_{i-1}\left(k_{i-1}\right) g\left(n_{i}, k_{i}-k_{i-1}, p\right) & i>1 \\
1 & i=1 .\end{cases}
\end{aligned}
$$

PROOF. The probability of continuing sampling at $i$-th stage is sum of conditional probabilities

$$
\sum_{k_{i}=C_{i-1}+1}^{C_{N}} h_{i-1}\left(k_{i}\right)
$$


based on cumulative binomial probability. Therefore, the power, ASN and AC are given by its functions.

\subsection{Repeated significance test}

Pocock [13] proposed an $N$ stage group repeated significance test of Type 1 with sample size $n$ for two sample normal mean test from normal populations $N\left(\mu_{i}, \sigma^{2}\right)$, $i=1,2, \sigma^{2}$ : known. He obtained equal nominal significance level $\alpha_{i}, i=1,2, \cdots, N$, for overall significance level $\alpha=0.050$ in testing the hypothesis $H_{0}: \mu_{1}=\mu_{2}$ against $H_{1}$ : $\mu_{1} \neq \mu_{2}$. The extended process adding different sample size $n_{m}$ at $m$-th stage, $m=$ $1,2, \cdots, N$, is shown in the following way.

Procedure 3. At $m$-th stage, $1 \leqq m \leqq N$,

$$
\begin{array}{lll}
\text { continue sampling, } & p_{m}>\alpha_{m} \text { and } m<N, \\
\text { accept } H_{0}, & \text { if } & p_{m}>\alpha_{m} \text { and } m=N, \\
\text { accept } H_{1}, & \text { if } & p_{m} \leqq \alpha_{m},
\end{array}
$$

where

$$
\begin{aligned}
& p_{m}=2\left[1-\Phi\left\{\sqrt{M_{m}} d_{m} / \sqrt{2} \sigma\right\}\right], \quad M_{m}=\sum_{j=1}^{m} n_{j}, \quad d_{m}=\left\{\sum_{j=1}^{m}\left(n_{j}\left(\bar{x}_{1 j}+\bar{x}_{2 j}\right)\right\} / M_{m},\right. \\
& \vec{x}_{i j}=\left[\sum_{k=1}^{n_{j}} x_{i j k}\right] / n_{j}, \quad \Phi(x)=\int_{-\infty}^{x} \phi(t) d t, \quad \phi(t)=\exp \left[-t^{2} / 2\right] /(\sqrt{2 \pi}), \\
& \alpha_{m} \text { : nominal significance level of } m \text {-th stage, }(i=1,2),(j=1,2, \cdots, N) .
\end{aligned}
$$

Then, the power, ASN and AC are given by the following Theorem 5 .

THEOREM 5. Let $T$ be an $N$ stage group repeated significance test, which has Procedure 3. Then, the power, $\mathrm{ASN}$ and $\mathrm{AC}$ are given by

$$
\begin{aligned}
& L(\mu)=1-\sum_{i=1}^{N} \int_{u\left(\alpha_{i} / 2\right)}^{\infty}\left[f_{i}(s)+f_{i}(-s)\right] d s, \\
& E_{1}(\mu)=2\left\{\sum_{i=1}^{N} M_{i} \int_{u\left(\alpha_{i} / 2\right)}^{\infty}\left[f_{i}(s)+f_{i}(-s)\right] d s+M_{N} \int_{-u\left(\alpha_{N} / 2\right)}^{u\left(\alpha_{N} / 2\right)} f_{N}(s) d s\right\}, \\
& E_{2}(\mu)=2 K_{1} \sum_{i=1}^{N-1} \int_{0}^{u\left(\alpha_{i} / 2\right)} f_{i}(s) d s+K_{2} E_{1}(\mu),
\end{aligned}
$$

respectively, where

$$
\begin{aligned}
& f_{i}(s)= \begin{cases}\phi\left(s-\delta_{1}\right) & (i=1) \\
\int_{0}^{u\left(\alpha_{i} / 2\right)} \phi\left(s-t-\delta_{i}\right) f_{i-1}(t) d t & (i=2,3, \cdots, N),\end{cases} \\
& \int_{0}^{u\left(\alpha_{0}\right)} f_{0}(s) d s=1, \quad \delta_{i}=\sqrt{n_{i}} \mu / \sigma, \quad \mu=\mu_{1}-\mu_{2} .
\end{aligned}
$$

Proof. The probability density function at $i$-th stage is given by the convolution

$$
\int_{0}^{u\left(\alpha_{i} / 2\right)} \phi\left(s-t-\delta_{i}\right) f_{i-1}(t) d t .
$$


Therefore the power, ASN and AC are given in use of this function, recursively.

COROLlary 2. Suppose that $T$ has Procedure 3 , then the overall significance level does not depend on sample size $n_{i}, i=1,2, \cdots, N$.

Proof. Under null hypothesis, $\mu_{1}=\mu_{2}, L(\mu)$ does not include $\delta_{i}$, i.e., sample size $n_{i}$.

Pocock obtained equal nominal significance levels $\alpha_{i}$ table for some stages. That is, the nominal significance levels are 0.029 and 0.016 for 2 and 5 stages when overall significance level is 0.050 , respectively. He recommended that these values are asymptotically adapted for normal mean test for unknown variance, i.e. $t$ test. For small sample size, however, $t$ test depends on sample size $n$. PRDIST command is to give easily such nominal probabilities. Table 3 shows a value of the final $\alpha_{N}$ in case of every $\alpha_{i}=0.029$ or 0.016 as Pocock suggested. Table 4 shows values of the final $\alpha_{N}$ in case of every $\alpha_{i}=0.010$ for overall $\alpha=0.050$. This shows that it is not so wrong to use the values of normal mean test for $t$ test. If sample size $n$ intends to infinity, final $\alpha_{N}$ equals to $\alpha_{i}$.

Gould-Pecore [7] has investigated about $N$ stage group repeated significance test of Type 2, with sample size $n$ for two sample normal mean test. For extended process adding different sample size $n_{m}$ at $m$-th stage, the following theorem is derived.

THEOREM 6 . Let $T$ be an $N$ stage group repeated significance test of Type 2 . Then, the power, ASN and $\mathrm{AC}$ are given by

$$
\begin{aligned}
L(\mu)= & 1-\sum_{i=1}^{N} \int_{u\left(\alpha_{i} / 2\right)}^{\infty}\left[g_{i}(s)+g_{i}(-s)\right] d s, \\
E_{1}(\mu)= & 2\left\{\sum_{i=1}^{N} M_{i}\left[\int_{u\left(\alpha_{i} / 2\right)}^{\infty}\left[g_{i}(s)+g_{i}(-s)\right] d s+\int_{-u\left(\alpha_{i A^{\prime} / 2}\right.}^{u\left(\alpha_{i A^{\prime}} / 2\right)} g_{i}(s) d s\right]\right. \\
& \left.+M_{N} \int_{-u\left(\alpha_{N A} / 2\right)}^{u\left(\alpha_{N} / 2\right)}\left[g_{N}(s)+g_{N}(-s)\right] d s\right\} \\
E_{2}(\mu)= & 2 K_{1} \sum_{i=1}^{N-1} \int_{u\left(\alpha_{\left.i_{A} / 2\right)}^{u\left(\alpha_{i} / 2\right)} g_{i}(s) d s+K_{2} E_{1}(\mu)\right.}
\end{aligned}
$$

where $\alpha_{m_{A}}$ : nominal acceptance probability at $m$-th stage,

$$
\begin{aligned}
& g_{i}(s)= \begin{cases}\phi\left(s-\delta_{1}\right) & (i=1) \\
\int_{0}^{u\left(\alpha_{i} / 2\right)} \phi\left(s-t-\delta_{i}\right) g_{i-1}(t) d t & (i=2,3, \cdots, N)\end{cases} \\
& \int_{0}^{u\left(\alpha_{0}\right)} g_{0}(s) d s=1, \quad \delta_{i}=\sqrt{n_{i}} \mu / \sigma, \quad \mu=\mu_{1}-\mu_{2}
\end{aligned}
$$

Proof. The probability density function at $i$-th stage is given

$$
\int_{0}^{u\left(\alpha_{i} / 2\right)} \phi\left(s-t-\delta_{i}\right) g_{i-1}(t) d t
$$

Therefore the power, ASN and AC are given similarly by Theorem 5 . 
Table 3 The final $\alpha_{N}$ in case of every sig. level is $\alpha_{i}=2.9 \%$ or $1.6 \%(i=1,2, \cdots, N-1)$ for $t$ test.

\begin{tabular}{|c|ccc|}
\hline $\mathrm{N}$ & 5 & 20 & 100 \\
\hline 2 & 0.027 & 0.029 & 0.029 \\
5 & 0.015 & 0.016 & 0.016 \\
\hline
\end{tabular}

Table 4 The final $\alpha_{N}$ in case of every sig. level is $\alpha_{i}=1.0 \%(i=1,2, \cdots, N-1)$ for $t$ test.

\begin{tabular}{|c|cc|}
\hline $\mathrm{N}$ & 5 & 20 \\
\hline 3 & 0.040 & 0.041 \\
5 & 0.033 & 0.034 \\
7 & 0.025 & 0.027 \\
\hline
\end{tabular}

Table 5 The final $\alpha_{N}$ in case of every nominal significance level is used $\alpha_{i}=3.1 \%$ or $2.4 \%(i=1,2, \cdots, N-1)$ for $t$ test for $\alpha_{i A}=50 \%$.

\begin{tabular}{|c|ccc|}
\hline $\mathrm{N}$ & 5 & 20 & 100 \\
\hline 2 & 0.028 & 0.031 & 0.031 \\
3 & 0.019 & 0.023 & 0.024 \\
\hline
\end{tabular}

Gould-Pecore obtained equal nominal significance levels $\alpha_{i}=0.031$ and 0.024 for 2 and 3 stages, respectively. Table 5 shows the final values $\alpha_{N}$ corrected for overall $\alpha=0.050$ for the probability of acceptance $\alpha_{i_{A}}=0.500$.

\section{Inference Processes via Different Tests with a Similar Purpose}

In view of practical and reasonable situations, there are some inference processes which are composed of two or three different nonparametric and parametric tests with a similar purpose. Elder-Muse [6] discussed a two stage process, including both tests by attributes with unit-limit and variables with average-limit. That is, let $T$ be a two stage test process with sample size $n$ from normal population $N\left(\mu, \sigma^{2}\right)$. Let $\mu_{0}$ denote the average limit, and let $\mu_{0}-c \sigma$ denote the unit limit and $k$ represent the maximum number of unit limit violations, where $c$ is an arbitrary positive constant.

THEOREM 7. Elder-Muse [6] Let $T$ be a two stage test process of Type 1, composed both tests by attributes with unit limit and variables with average limit. Then, the power is given by

$$
L(\mu)=1-\sum_{i=0}^{k}\left(\begin{array}{c}
n \\
i
\end{array}\right)[\Phi(c-t)]^{n-i}[\Phi(t-c)]^{i}\left\{1-\Phi\left[\left(\mu_{0}-a(t)\right) / \sqrt{b(t)}\right]\right\},
$$

where

$$
a(t)=\mu+\sigma g(v)(n-i) / n-\sigma g(-v) i / n,
$$




$$
\begin{aligned}
& b(t)=\left(\sigma^{2} / 2\right)(1-g(v)(v+g(v))(n-i) / n-g(-v)(-v+g(-v)) i / n), \\
& g(v)=\phi(v) / \Phi(v), \quad v=c-t, \quad t=\left(\mu_{0}-\mu\right) / \sigma .
\end{aligned}
$$

PROOF. The probability of acceptance is given by the product of conditional probability derived using truncated normal probability density functions and the probability of $i$-th limit violations.

For another process of Type 1 for location, median, Wilcoxon and $t$ tests in order are applied. That is, three stage group sampling process of Type 1 , composed median, Wilcoxon and $t$ tests adding sample $n_{i}, i=1,2,3$, are to be considered. In these cases, PRTP command is applicable to study statistical properties of the processes. For instance, the overall significance level is shown 0.0910 , that is, 0.0226 for median test, 0.0394 for Wilcoxon test, 0.0290 for $t$ test, to an exponential distribution with mean zero and variance unity, where $n_{1}=n_{2}=n_{3}=10$ and $\alpha_{1}=\alpha_{2}=\alpha_{3}=0.050$.

\section{Inference Processes via Different Tests with Different Purposes}

The inference processes in this section are mainly used for composite hypothesis and series of hypotheses, in which some different statistical tests are applied by not adding samples, for example, location and dispersion tests for the type of distribution and multiple test. The former process is used for testing a hypothesis $H_{0}: F(x)=F_{0}(x)$ against $H_{1}: F(x)=F_{1}(x)$. The power of this process becomes complicated, and alternative distribution may be skewed or unclear types of distribution. The illustrative hypothesis is $H_{0}: \mu=\mu_{0}, \sigma^{2}=\sigma_{0}^{2}$ against $H_{1}: \mu \neq \mu_{0}, \sigma^{2} \neq \sigma_{0}^{2}$. The latter process is used for testing the series of hypotheses $H_{01}, H_{02}, \cdots, H_{0 N}$ against hypotheses $H_{11}, H_{12}, \cdots, H_{1 N}$. The simple process may be two stage process with chi-square test for dispersion and $u$-test for mean. The first stage is whether the variance is unity, i.e., $\sigma^{2}=1$ or not, if accepted, and secondly to test whether mean is zero or not under the condition $\sigma^{2}=1$.

THEOREM 8. Let $T$ be two stage process of Type 1 with chi-square test for dispersion and u-test for mean. Then, the mathematical formulation for power is shown by

$$
L(\mu)=C \int_{0}^{\chi_{n-1}^{2}\left(\alpha_{1}\right) / \lambda} \int_{-\infty}^{u\left(\alpha_{2}\right)-\imath \bar{n} \mu} x^{(n-3) / 2} \exp \left[-\left(x+u^{2}\right) / 2\right] d u d x,
$$

where

$$
C=2^{-n / 2}\{\sqrt{\pi} \Gamma[(n-1) / 2]\}^{-1} .
$$

Proof. The joint distribution of $\chi_{n-1}^{2}$ and $u$ distributions is shown by

$$
\left.2^{-n / 2}\{\sqrt{\pi} \Gamma[(n-1) / 2]\}^{-1} x^{(n-3) / 2} \exp \left[-\left(x+u^{2}\right) / 2\right]\right) .
$$

Therefore, the power function is given by (5.1).

The illustrative nonparametric two stage process of Type 1 is Ansari-Bradley test for dispersion and Wilcoxon test for location in order, which are independent tests each other. This process is examined to calculate nominal $\alpha_{i}, i=1,2$, for fixed overall $\alpha$ by PRDIST command. Given $\alpha_{1}=0.050$ and overall $\alpha=0.100$, the percent point for $\alpha_{2}$ is given by about 1.94 for both sides test. This value is corresponding to 0.0524 for both side probabilities under normal distribution, where theoretical value calculated from Theorem 1 is 0.0526 . Note that the properties of this test process are different from 
those of Lepage test, whose statistic is shown by the sum of square of these of these two statistics.

For extended another process, an inference process which consists of test for the type of distribution and the local most powerful test for the prespecified distribution is to be considered. The test for normal distribution and $t$ test, the test for logistic distribution and Wilcoxon test and the test for exponential distribution and Savage test, in order, are illustrative test processes.

\section{Extension of Relative Efficiency to Inference Process and Discussion}

Comparing two techniques for the inference, efficiency and deficiency are usually used. These values are calculated by the ratio of samples or variances of two techniques which achieve the prefixed design. In case of two tests, the relative efficiency is obtained by the ratio of sample sizes of two tests with just equal power for the same design. The asymptotic relative efficiency also obtained by the limiting ratio of sample sizes required to the limiting power. That is, the relative efficiency and asymptotic relative efficiency between two tests are defined in the following way. Consider samples from distribution $F(x \mid \theta)$ and let $T_{1}$ and $T_{2}$ be two tests with given level of significance levels $\alpha$ for the hypothesis $H_{0}: \theta=\theta_{0}$ against the alternative $H_{1}: \theta=\theta_{1}$. Let $\beta_{N}(\theta)$ and $\beta_{N}^{*}(\theta)$ denote the power functions of $T_{1}$ and $T_{2}$ based on $N$ observations.

Definition 2. Relative efficiency (Lehmann [9]) Let $\beta_{N_{1}}(\theta)$ be a specificd power given $\theta$ and $N 1$ with $\alpha<\beta<1$. Then, if there exists $N 2$ such that $\beta_{N_{2}}^{*}(\theta)=\beta_{N_{1}}(\theta)$,

$$
\rho\left(T_{1}, T_{2}\right)=N 1 / N 2
$$

is defined to be the relative efficiency of the test $T_{2}$ with respect to the test $T_{1}$.

Definition 3. Asymptotic relative efficiency (Pitman [12]) Let $\beta_{N}(\theta)$ be a specified power given $\theta$ and $N$ with $\alpha<\beta<1$. Consider a sequence of alternatives $\theta_{N}$ such that $\beta_{N}\left(\theta_{N}\right) \rightarrow \beta_{0}($ as $N \rightarrow \infty)$, and a sequence $M=h(N)$ such that $\beta_{M}^{*}\left(\theta_{N}\right) \rightarrow \beta_{0}$ (as $N \rightarrow \infty$ ), where $\beta_{0}$ is a constant value which belongs to $(0,1)$. Then if

$$
e\left(T_{1}, T_{2}\right)=\lim _{N \rightarrow \infty} N / M
$$

exists and is independent of $\alpha$ and $\beta, e\left(T_{1}, T_{2}\right)$ is defined to be the asymptotic relative efficiency of the test $T_{2}$ with respect to the test $T_{1}$.

For the relative efficiency, the following theorem is derived.

THEOREM 9. Let $\rho\left(T_{i}, T_{j}\right)$ be the relative efficiency of test $T_{j}$ with respect to the test $T_{i}, i, j=1,2,3, i \neq j$. For a specified power given $\theta$,

$$
\begin{aligned}
& \rho\left(T_{i}, T_{j}\right)=1 / \rho\left(T_{j}, T_{i}\right), \\
& \rho\left(T_{i}, T_{k}\right)=\rho\left(T_{i}, T_{j}\right) \rho\left(T_{j}, T_{k}\right),
\end{aligned}
$$

where $i, j, k=1,2,3, i \neq j, j \neq h, i \neq k$.

PROOF. Proof is omitted.

The extended relative efficiency is defined to the test process $T_{2}$ with respect to the test $T_{1}$. 
Table 6 The relative efficiency of two stage $t$ test with respect to $t$ test.

\begin{tabular}{|c|ccccc|}
\hline mean & 0.2 & 0.4 & 0.6 & 0.8 & 1.0 \\
\hline efficiency & 0.810 & 0.897 & 0.912 & 0.966 & 1.123 \\
\hline
\end{tabular}

Definition 4. Let $\beta_{N}(\theta)$ be a specified power given $\theta$ and $N$ with $\alpha<\beta<1$. Then if there exists $\mathrm{ASN}\left(T_{2}\right)$ such that $\beta_{\mathrm{ASN}\left(T_{2}\right)}(\theta)=\beta_{N}(\theta)$,

$$
\rho\left(T_{1}, T_{2}\right)=N / \operatorname{ASN}\left(T_{2}\right)
$$

is defined to be the relative efficiency of the test process $T_{2}$ with respect to the test $T_{1}$.

The numerical evaluation is applied for relative efficiency of two stage $t$ test process $T_{2}$, which has same additional sample numbers, with respect to ordinary $t$ test $T_{1}$. Table 6 shows the values of relative efficiency on normal distribution with mean zero and variance unity for the first population, and the second population is shifted with the same variance and distribution type, where sample sizes are $n_{1}=n_{2}=15$ for the test process $T_{2}$ with respect to the test $T_{1}$.

\section{Conclusion}

Although there are difficulties and complications to make clear properties numerically by the theoretical formulae under variety of conditions except only the simple case like in statistical text book, there exist much more possibilities in view of statistical computing to evaluate statistical properties under various situations. Actually, the knowledge commands in NISAN system provides useful knowledge, which makes clear the properties numerically under variety of conditions in statistical data analysis.

\section{Acknowledgement}

The author would like to express his sincere gratitude to Professor Chooichiro Asano of Kyushu University for his valuable suggestions, constructive comments and continuous encouragement.

\section{References}

[1] Aroian, L.A.: Sequential analysis, Direct method, Technometrics, 10 (1968), 125-132.

[2] Asavo, Ch., Jojima, K., Kurihara, K. and Ichimura, M.: Developmental studies and evaluation of sampling inspection plans on the basis of a software system, Frontiers in Statistical Quality Control, Physica-Verlag, (1984), 107-120.

[3] Asano, Ch., Kurihara, K. and Geng, Z.: Sequential inference processes in computational aspects, Proceedings in Computational Statist., Physica-Verlag, (1986), 63-68.

[4] Colton, T. and McPherson, K.: Two-stage plans compared with fixed sample size and Wald SPRT plans, Jour. Amer. Statist. Assoc., 71 (1976), 80-86.

[5] EEden, C.: The relation between Pitman's asymptotic relative efficiency of two tests and the correlation coefficient between their test statistics, Ann. Math. Statist., (1963), 14421451.

[6] ELDER, R.S. and MUSE, H.D.: An approximate method for evaluating mixed sampling 
plans, Technometrics, 24 (1982), 207-211.

[7. Golld, A.L. and PECORE, V.J.: Group sequential methods for clinical trials allowing early acceptance of $H_{0}$ and incorporating costs, Biometrika, 69 (1982), 75-80.

[8] Kitag.aw. T.: Successive process of statistical inference, Bull. Math. Statist. 5 (1952), $35-50$.

[9] Lehman, E. and D'Abrera, H.J.M.: Nonparametrics, Holden-Day, (1975).

[10] McPherson, C.K. and Armitage, P.: Repeated significance tests on accumulating data when the null hypothesis is not true, J. Roy. Statist. Soc. Ser. A, (1971), 15-26.

[11] Miller, R.G.Jr.: Simultaneous statistical inference, Mcgraw-Hill, New York, (1966).

[12] PItman, E. J.E.: Lecture notes on nonparametric statistical inference, Columbia University, (1949).

[13] Рососк, S.J.: Group sequential methods in the design and analysis of clinical trials, Biometrika, 64 (1977), 191-199.

[14] WaLd, A.: Sequential analysis, John Wiley \& Sons (1947).

Received October 2, 1987

Communicated by Ch. Asano 